\title{
Unitarity constraints on ALP interactions
}

\author{
I. Brivio ${ }^{*}$ \\ Institut für Theoretische Physik, Universität Heidelberg. \\ Philosophenweg 16, 69120 Heidelberg (Germany) \\ O. J. P. Éboli॰ ${ }^{\dagger}$ \\ Instituto de Física, Universidade de São Paulo, São Paulo-SP 05508-090, Brazil \\ M. C. González-García $\odot^{\ddagger}$ \\ Institució Catalana de Recerca i Estudis Avançats (ICREA), \\ Departament d'Estructura i Constituents de la Matèria, Universitat de Barcelona, \\ 647 Diagonal, E-08028 Barcelona, Spain \\ and C.N. Yang Institute for Theoretical Physics, SUNY at Stony Brook, \\ Stony Brook, New York 11794-3840, USA
}

(Received 25 June 2021; accepted 27 July 2021; published 27 August 2021)

\begin{abstract}
We derive partial-wave unitarity constraints on gauge-invariant interactions of an axionlike particle up to dimension-6 from all allowed $2 \rightarrow 2$ scattering processes in the limit of large center-of-mass energy. We find that the strongest bounds stem from scattering amplitudes with one external axionlike particle and only apply to the coupling to a pair of $S U(2)_{L}$ gauge bosons. Couplings to $U(1)_{Y}$ and $S U(3)_{C}$ gauge bosons and to fermions are more loosely constrained.
\end{abstract}

DOI: 10.1103/PhysRevD.104.035027

\section{INTRODUCTION}

Axionlike particles (ALPs) are generic pseudoGoldstone bosons that can emerge from the spontaneous breaking of some global symmetry at energies well above the electroweak (EW) scale $v$. While the main representative is the QCD axion (either "invisible" [1-8] or in modern setups where the tie between axion mass and couplings is relaxed [9-27]), this class of particles encompasses a large number of exotic states that can emerge in composite Higgs models [28-32], models with spontaneous breaking of lepton number ("majorons") [33,34], dynamical flavor theories ("axiflavons") [35-38], string theory $[39,40]$, and many other scenarios.

ALPs are usually studied within a model-independent effective field theory (EFT) framework [41,42]. Their pseudo-Goldstone nature justifies the assumption that ALPs are the only light remnant of a much heavier new physics sector, whose interactions are suppressed by a

\footnotetext{
*brivio@thphys.uni-heidelberg.de †eboli@if.usp.br

"maria.gonzalez-garcia@stonybrook.edu
}

Published by the American Physical Society under the terms of the Creative Commons Attribution 4.0 International license. Further distribution of this work must maintain attribution to the author(s) and the published article's title, journal citation, and DOI. Funded by SCOAP . characteristic scale $f_{a} \gg v$. A priori, the allowed parameter space spans several orders of magnitude both in the ALP mass $m_{a}$ and in the couplings to Standard Model (SM) particles that, within the EFT approach, enter at lowest order as dimension-5 operators.

The interest in the ALP Lagrangian as a self-consistent EFT has grown recently, leading to several studies of its theoretical properties. For instance, the renormalization group evolution and the matching to the ALP EFT valid below the EW scale were derived in Refs. [43-45]. The interplay between dimension-5 ALP interactions and dimension-6 operators in the Standard Model EFT (SMEFT) was explored in Ref. [46]. The matching of the ALP EFT to concrete QCD axion models was examined in Ref. [47], which pointed out theoretical subtleties when applying the EFT approach to loop processes.

In this work, we examine the validity range of the ALP EFT at high energies on the basis of its perturbative partialwave unitarity properties. It is well known that classically nonrenormalizable interactions give rise to rapid growth of the scattering amplitudes with energy, which leads to partial-wave unitarity violation at some large value of the center-of-mass energy $\sqrt{S}$. Generically, partial-wave unitarity violation signals the breakdown of the low-energy description and indicates that extra fundamental degrees of freedom or the onset of the nonperturbative regime must be present around or below the apparent unitarity violation 
scale in order to restore the physical behavior of scattering amplitudes. Paradigmatic examples of the use of unitarity relations to derive constraints on the validity of a theory include the seminal work of Lee et al. $[48,49]$ that imposed an upper bound on the Higgs mass by analyzing the unitarity of the standard model and was used to build a case in favor of the construction of the present generation of colliders. Another classical example are the bounds on new fermions obtained by Chanowitz et al. [50]. On a more formal front, unitarity arguments have also been employed, for example, in connection with the requirement of gauge invariance [51].

In the last decades, partial-wave unitarity has been employed ubiquitously to constrain effective interactions, in particular in the electroweak sector (see, for example, Refs. [52-59]). Recently, Refs. [60-62] presented a general systematic study of unitarity bounds for the case of effective interactions in the SMEFT and Higgs EFT (HEFT). Generically, unitarity preservation imposes consistency conditions on the theory such that, for the EFT to be valid up to a given $\sqrt{S}$, the effective couplings (scale) need to be smaller (larger) than a certain threshold. Conversely, for given values of the EFT coefficients and scale, unitarity imposes an upper limit on the energy scales at which the EFT can be applied. In that respect, unitarity bounds are crucial for the interpretation of actual experiments, which study tails of kinematical distributions, since one can infer unphysical bounds that are too strong if these limits are not respected.

For the case of ALP EFT, the rapid growth of the scattering amplitudes with energy, which leads to partialwave unitarity violation, is particularly enhanced. The reason for that is the pseudo-Goldstone nature of the ALPs which requires all their interactions to be classically invariant under shifts $a(x) \mapsto a(x)+\alpha$, i.e., to be of the form $J^{\mu} \partial_{\mu} a$. As a consequence, an explicit momentum dependence is present in all ALP couplings.

A partial analysis of unitarity constraints on ALP couplings was presented in Refs. [63,64]. Here we adopt a more systematic approach and derive maximal constraints on all ALP interactions of dimension 5 and 6 from partial-wave unitarity, examining all allowed $2 \rightarrow 2$ scattering processes in the limit of large center-of-mass energy. We adopt a procedure analogous to the one employed in [60-62] for the case of effective interactions in the SMEFT and HEFT.

The outline of this article is as follows. We present the relevant Lagrangian employed in Sec. II and briefly discuss the number of relevant operators we consider. The core of the results is contained in Sec. III, where we derive first the most general bounds for the ALP couplings to SM gauge bosons which are obtained from the partial-wave analysis of the scattering of boson pairs in Sec. III A. Section III B contains our derivation of the most general independent constraints on ALP couplings to SM fermions which are obtained with the partial-wave analysis of scatterings involving fermion pairs. We briefly discuss the results in Sec. IV. Explicit expressions of the helicity amplitudes for all the relevant processes are presented in the Appendix.

\section{ALP EFFECTIVE LAGRANGIAN}

We consider the SM extended by the ALP effective Lagrangian $[41,42,65]$

$$
\begin{aligned}
\mathcal{L}_{A L P}= & \frac{1}{2} \partial_{\mu} a \partial^{\mu} a-\frac{m_{a}^{2}}{2} a^{2}+C_{\tilde{B}} O_{\tilde{B}}+C_{\tilde{W}} O_{\tilde{W}} \\
& +C_{\tilde{G}} O_{\tilde{G}}+C_{a \Phi} O_{a \Phi} \\
& +\left[C_{u \Phi} O_{u \Phi}+C_{d \Phi} O_{d \Phi}+C_{e \Phi} O_{e \Phi}+\text { H.c. }\right] \\
& +C_{a \Phi}^{(2)} O_{a \Phi}^{(2)},
\end{aligned}
$$

where the effective operators

$$
\begin{gathered}
O_{\tilde{B}}=\frac{a}{f_{a}} B_{\mu \nu} \tilde{B}^{\mu \nu}, \quad O_{u \Phi}=i \frac{a}{f_{a}} \bar{q} Y_{u} \tilde{\Phi} u, \\
O_{\tilde{W}}=\frac{a}{f_{a}} W_{\mu \nu}^{i} \tilde{W}^{i \mu \nu}, \quad O_{d \Phi}=i \frac{a}{f_{a}} \bar{q} Y_{d} \Phi d, \\
O_{\tilde{G}}=\frac{a}{f_{a}} G_{\mu \nu}^{a} \tilde{G}^{a \mu \nu}, \quad O_{e \Phi}=i \frac{a}{f_{a}} \bar{l} Y_{e} \Phi e, \\
O_{a \Phi}=\frac{\partial_{\mu} a}{f_{a}}\left(i \Phi^{\dagger}\left(D_{\mu} \Phi\right)-i\left(D_{\mu} \Phi\right)^{\dagger} \Phi\right) \\
O_{a \Phi}^{(2)}=\frac{\partial_{\mu} a \partial^{\mu} a}{f_{a}^{2}}\left(\Phi^{\dagger} \Phi\right)
\end{gathered}
$$

form a complete basis of $C P$-even ALP interactions up to $\mathcal{O}\left(f_{a}^{-3}\right)$ terms. Here, $B_{\mu}, W_{\mu}^{i}$, and $G_{\mu}^{a}$ are the gauge bosons of the $U(1)_{Y} \times S U(2)_{L} \times S U(3)_{c}$ SM symmetry, respectively, and the dual field strengths are defined by $\tilde{X}_{\mu \nu}=\frac{1}{2} \varepsilon_{\mu \nu \rho \sigma} X^{\rho \sigma} . \Phi$ denotes the $S U(2)_{L}$ Higgs doublet, while $\tilde{\Phi}=i \tau^{2} \Phi^{*}$ is its dual (with $\tau^{i}$ being the Pauli matrices). Upon EW symmetry breaking, $\left\langle\Phi^{\dagger} \Phi\right\rangle=$ $(v+H)^{2} / 2$, with $H$ being the physical Higgs boson. The left- (right-)handed fermion multiplets are denoted by $q, l(u, d, e)$, and $Y_{u}, Y_{d}, Y_{e}$ are the $3 \times 3$ Yukawa matrices. All index contractions were left implicit, and repeated indices are summed over unless otherwise specified. A mass term $m_{a}$ for the ALP was introduced, which is generically induced in the presence of soft breaking of shift invariance, such as nonperturbative instanton effects in the case of the QCD axion [6,66-68].

We neglect $C P$-violating effects such that all Wilson coefficients $C_{i}$ are real scalar quantities. Although this is 
not manifest in Eqs. (2)-(5), all ALP interactions are classically shift invariant: the interactions to bosons can be written as $\partial_{\mu} a J_{X}^{\mu}$ by integration by parts, where $J_{X}^{\mu}$ is the Chern-Simons current associated to the $X=\{B, W, G\}$ gauge boson. ${ }^{1}$ The operators with fermions were taken to follow the minimal-flavor-violation ansatz [69-71], i.e., to respect a $U(3)^{5}$ global symmetry that is only broken by insertions of the Yukawa couplings. With this flavor structure, they could also be equivalently traded for a set of chirality-conserving ones of the form $\left(\partial_{\mu} a\right)\left(\bar{\psi}_{p} \gamma^{\mu} \psi_{r}\right) \delta^{p r}$, with $p, r$ flavor indices [44,72].

The operator $O_{a \Phi}$ is actually redundant $[41,45,65,72]$ :

$$
O_{a \Phi}=O_{u \Phi}-O_{d \Phi}-O_{e \Phi}+\text { H.c. }
$$

Nevertheless, it is often retained because the set $\left\{O_{\tilde{B}}, O_{\tilde{W}}, O_{\tilde{G}}, O_{a \Phi}\right\}$ forms a complete and nonredundant operator basis at dimension 5 in the bosonic sector that can be of phenomenological interest.

The operator $O_{a \Phi}^{(2)}$ has been previously considered in Refs. [73-76], and it is the only shift-invariant operator $^{2}$ that can be constructed at dimension 6 . SMEFT operators of dimension 6 are neglected; we assume them to be suppressed by a scale $\Lambda_{\mathrm{SMEFT}} \neq f_{a}$ and work consistently at order $\left(f_{a}^{-2} \Lambda_{\mathrm{SMEFT}}^{0}\right)$. Discussing the interplay of the two expansions is beyond the scope of this work. ${ }^{3}$

\section{ANALYSIS OF UNITARITY CONSTRAINTS}

\section{A. Helicity amplitudes for the scattering of pairs of bosons}

Consider the two-to-two scattering of bosons $V_{i}$ with helicities $\lambda_{i}$,

$$
V_{1 \lambda_{1}} V_{2 \lambda_{2}} \rightarrow V_{3 \lambda_{3}} V_{4 \lambda_{4}}
$$

\footnotetext{
${ }^{1}$ In the $G$ case, only a discrete version of the shift invariance is preserved due to the presence of nonvanishing instanton configurations.

${ }^{2}$ One more operator structure is present at dimension 6, namely, $\left(\partial_{\mu} \partial^{\mu} a\right)^{2}$. However, applying the ALP equation of motion, this can be fully reabsorbed into a redefinition of the ALP mass. We have checked the completeness of the dimension6 set with BASISGEN [77].

${ }^{3}$ As will become clear from the discussion in Sec. III, the bounds on $C_{\tilde{W}}, C_{f \Phi}, C_{a \Phi}$, and $C_{a \Phi}^{(2)}$ are not expected to change significantly in the presence of dimension-6 SMEFT operators, independently of the interplay between the SMEFT and ALP expansions. This is because all these bounds are dominated by scatterings with one or two external ALPs.
}

where we denote by $V$ either gauge bosons, Higgs, or ALP. The corresponding helicity amplitude can be expanded in partial waves in the center-of-mass system as [78]

$$
\begin{aligned}
& \mathcal{M}\left(V_{1 \lambda_{1}} V_{2 \lambda_{2}} \rightarrow V_{3 \lambda_{3}} V_{4 \lambda_{4}}\right) \\
&= 16 \pi \sum_{J}(2 J+1) \sqrt{1+\delta_{V_{1 \lambda_{1}}}^{V_{2 \lambda_{2}}}} \sqrt{1+\delta_{V_{3 \lambda_{3}}}^{V_{4 \lambda_{4}}}} \\
& \quad \times d_{\lambda \mu}^{J}(\theta) e^{i M \varphi} T^{J}\left(V_{1 \lambda_{1}} V_{2 \lambda_{2}} \rightarrow V_{3 \lambda_{3}} V_{4 \lambda_{4}}\right),
\end{aligned}
$$

where $\lambda=\lambda_{1}-\lambda_{2}, \mu=\lambda_{3}-\lambda_{4}, \quad M=\lambda_{1}-\lambda_{2}-\lambda_{3}+\lambda_{4}$, and $\theta(\varphi)$ is the polar (azimuthal) scattering angle. $d$ is the usual Wigner rotation matrix. This expression holds for gauge bosons with $\lambda=0, \pm 1$ and for scalars (Higgs or ALP) with $\lambda \equiv 0$; the fermion case will be addressed below. For further details and conventions, see Ref. [60].

In the limit $S \gg\left(M_{V_{1}}+M_{V_{2}}\right)^{2}$, partial-wave unitarity for a given elastic channel requires that

$$
\left|T^{J}\left(V_{1 \lambda_{1}} V_{2 \lambda_{2}} \rightarrow V_{1 \lambda_{1}} V_{2 \lambda_{2}}\right)\right| \leq 1
$$

The most stringent bounds are obtained by diagonalizing $T^{J}$ in the particle and helicity space and then applying the condition in Eq. (9) to each of the eigenvalues. This is the approach which we follow.

We start by calculating the scattering amplitudes for all possible combinations of bosons and helicities generated by the SM extended with the Lagrangian in Eq. (1) for a given total electric charge $Q=2,1,0$ and that give nonvanishing projections on a given partial-wave $J$ proportional to some ALP coupling. Conservation of color implies that initial or final states with color have to be considered independently of those in a color singlet state. So, one is led to consider separately the $T^{J}\left(\bar{T}^{J}\right)$ amplitude matrices for processes with color singlet (octet) in the initial and final states. One must also take into account that parity conservation at tree level implies the relation

$$
\begin{aligned}
& T^{J}\left(V_{1 \lambda_{1}} V_{2 \lambda_{2}} \rightarrow V_{3 \lambda_{3}} V_{4 \lambda_{4}}\right) \\
& \quad=(-1)^{\lambda_{1}-\lambda_{2}-\lambda_{3}+\lambda_{4}} T^{J}\left(V_{1-\lambda_{1}} V_{2-\lambda_{2}} \rightarrow V_{3-\lambda_{3}} V_{4-\lambda_{4}}\right)
\end{aligned}
$$

and leads to a reduction of the number of independent helicity amplitudes. Time-reversal invariance further reduces the number of helicity amplitudes that need to be evaluated.

Altogether, the initial/final states contributing a priori to the $T^{J}$ matrices for each value of $Q$ and $J$ are 


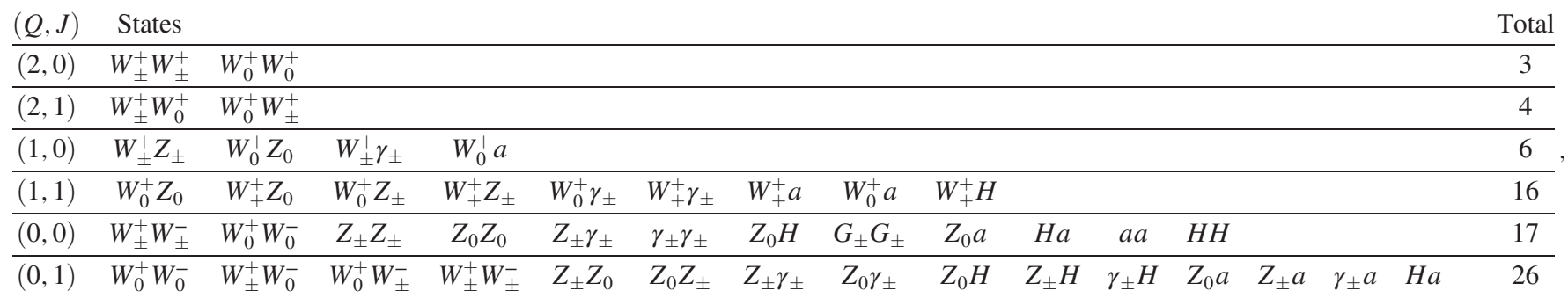

and correspondingly the states contributing to the $\bar{T}^{J}$ matrices are

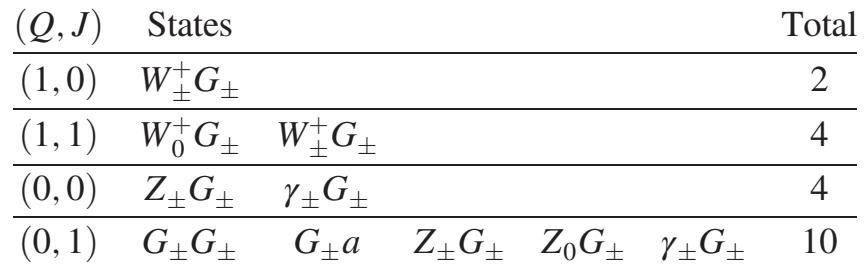

where upper indices indicate charge and lower indices helicity. We also display in Eqs. (11) and (12) the dimensionality of the particle and helicity matrix for each independent $(Q, J)$ channel. In Eq. (11), the states $H H$ and $W^{ \pm} H$ are only present when the dimension- 6 operator is considered.

We list in Tables I-IV, which are in the Appendix, the expressions for the most $S$-divergent part of the amplitudes for the channels which give the dominant contribution to the $T^{J}$ and $\bar{T}^{J}$ matrices.

\section{Bounds on individual operators}

As seen in Tables I-III, for processes with zero or two ALPs as external states, the most energy-divergent amplitudes occur for scattering of transversely polarized gauge bosons, as expected. These amplitudes are all proportional to the product of two axion couplings; therefore, the two powers of their momentum involved in the coupling of ALP to the gauge boson generate the leading $S / f_{a}^{2}$ dependence. A good fraction of them contributes to $J=0$ matrices, which are, a priori, expected to lead the strongest bounds. Furthermore, for amplitudes with gluon pairs, the strongest bounds are obtained for the gluon pair in the singlet color state $\frac{1}{N_{C}^{2}-1} \sum_{a=1}^{N_{C}^{2}-1}\left|G^{a} G^{a}\right\rangle$.

Altogether from the diagonalization of the $J=0$ matrices and assuming only one nonzero coupling at a time, we find that the largest eigenvalues correspond to the $Q=0$, $T^{0}$ matrix and read

$$
\begin{array}{lr}
\frac{1+\sqrt{97}}{16 \pi} \frac{S}{f_{a}^{2}} C_{\tilde{W}}^{2}, & \frac{1+\sqrt{33}}{16 \pi} \frac{S}{f_{a}^{2}} C_{\tilde{B}}^{2}, \\
\frac{4\left(N_{C}^{2}-1\right)}{\pi} \frac{S}{f_{a}^{2}} C_{\tilde{G}}^{2} & \text { and } \quad \frac{1}{32 \pi} \frac{S}{f_{a}^{2}} C_{a \Phi}^{(2)},
\end{array}
$$

respectively. Applying the condition in Eq. (9) to each of these eigenvalues, we obtain the bounds

$$
\begin{aligned}
\left|C_{\tilde{W}}\right| & \leq 2.1 \frac{f_{a}}{\mathrm{TeV}}\left(\frac{\mathrm{TeV}}{\sqrt{S}}\right), \\
\left|C_{\tilde{B}}\right| & \leq 2.7 \frac{f_{a}}{\mathrm{TeV}}\left(\frac{\mathrm{TeV}}{\sqrt{S}}\right), \\
\left|C_{\tilde{G}}\right| & \leq 0.31 \frac{f_{a}}{\mathrm{TeV}}\left(\frac{\mathrm{TeV}}{\sqrt{S}}\right), \\
\left|C_{a \Phi}^{(2)}\right| & \leq 101 \frac{f_{a}^{2}}{\mathrm{TeV}^{2}}\left(\frac{\mathrm{TeV}}{S}\right) .
\end{aligned}
$$

We observe that the constraint on the dimension-6 operator $O_{a \Phi}^{(2)}$ is dominated by scattering amplitudes with two ALP external states.

Unlike the amplitudes with an even number of ALP in the external states, some helicity amplitudes with only one ALP in either the initial or final state have a leading behavior $S^{\frac{3}{2}} /\left(f_{a} M_{W}^{2}\right)$, as seen in Table IV. ${ }^{4}$ These amplitudes involve two longitudinally polarized gauge bosons, whose polarization vectors are proportional to $\sqrt{S}$, and one transversely polarized gauge boson, whose momentum contributes another power of $\sqrt{S}$. This configuration can only be generated by a combination of the SM vertices and those induced by $O_{\tilde{W}}$, and, consequently, the amplitudes involve a single power of the $C_{\tilde{W}}$ coupling and of the SM coupling $e$ and do not depend on any other Wilson coefficient. As seen from the scattering angle dependence of the amplitudes in Table IV, they contribute only to the $T^{J=1}$ matrix with either $Q=0$ or $Q=1$. Diagonalizing these, we find that the largest eigenvalue is

$$
\frac{\sqrt{1+c_{w}^{2}+2 c_{w}^{4}}}{24 \pi} \frac{S^{3 / 2}}{f_{a} M_{W}^{2}} e C_{\tilde{W}},
$$

\footnotetext{
${ }^{4}$ This is in contrast with what is found for effective interactions in the SMEFT [60-62] for which all operators of a given dimension lead to most divergent amplitudes with the same power of $S$, that is, $S$ for dimension-6 operators and $S^{2}$ for dimension-8 operators.
} 
where $c_{w}$ is the cosine of the weak mixing angle. Therefore, the condition in Eq. (9) implies the constraint on $C_{\tilde{W}}$,

$$
\left|C_{\tilde{W}}\right| \leq 0.14 \frac{f_{a}}{\mathrm{TeV}}\left(\frac{\mathrm{TeV}}{\sqrt{S}}\right)^{3} .
$$

Comparing the bounds on $C_{\tilde{W}}$ from $J=0$-wave unitarity, Eq. (14), and from $J=1$-wave unitarity, Eq. (19), we find that the constraint derived from the $J=1$ amplitudes is the strongest for

$$
\sqrt{S}>260 \mathrm{GeV}
$$

\section{Including multiple operators simultaneously}

Fixing $C_{a \Phi}^{(2)}=0$ (or equivalently barring cancellations between dimension-5 and- 6 terms) and allowing multiple dimension-5 operators to vary simultaneously does not alter significantly the bounds reported above. For $C_{\tilde{W}}$, this is obvious because the leading constraint Eq. (19) is genuinely independent of the other Wilson coefficients. For $C_{\tilde{B}}$ and $C_{\tilde{G}}$, this can be understood by considering that $C_{\tilde{G}}$ is dominantly constrained by $G_{ \pm} G_{ \pm} \rightarrow G_{ \pm} G_{ \pm}$scattering in the color-singlet channel, which is independent of $C_{\tilde{B}}$. We have also verified this explicitly by diagonalizing the $Q=0 T^{J=0}$ matrix with $C_{\tilde{B}}, C_{\tilde{G}}$ present at the same time. The diagonalization can still be done analytically, though the resulting expressions for the eigenvalues are not particularly illuminating. Imposing the unitarity limits on those eigenvalues yields the same bounds as in Eqs. (15) and (16).

Allowing all operators of dimension 5 and 6 to be present simultaneously (i.e., allowing cancellations between both orders), we find that the largest eigenvalues are

$$
\begin{aligned}
& \frac{5}{8 \pi} \frac{S}{f_{a}^{2}} C_{\tilde{W}}^{2}, \quad \frac{1}{8 \pi} \frac{S}{f_{a}^{2}} C_{\tilde{B}}^{2}, \quad \frac{4\left(N_{C}^{2}-1\right)}{\pi} \frac{S}{f_{a}^{2}} C_{\tilde{G}}^{2} \\
& \text { and } \frac{1}{32 \pi} \frac{S}{f_{a}^{2}} C_{a \Phi}^{(2)},
\end{aligned}
$$

and correspondingly the unitarity limits on the Wilson coefficients are

$$
\begin{aligned}
\left|C_{\tilde{W}}\right| & \leq 2.2 \frac{f_{a}}{\mathrm{TeV}}\left(\frac{\mathrm{TeV}}{\sqrt{S}}\right), \\
\left|C_{\tilde{B}}\right| & \leq 5.0 \frac{f_{a}}{\mathrm{TeV}}\left(\frac{\mathrm{TeV}}{\sqrt{S}}\right), \\
\left|C_{\tilde{G}}\right| & \leq 0.31 \frac{f_{a}}{\mathrm{TeV}}\left(\frac{\mathrm{TeV}}{\sqrt{S}}\right), \\
\left|C_{a \Phi}^{(2)}\right| & \leq 101 \frac{f_{a}^{2}}{\mathrm{TeV}^{2}}\left(\frac{\mathrm{TeV}^{2}}{S}\right) .
\end{aligned}
$$

These results hold irrespective of whether $C_{\tilde{W}}, C_{\tilde{B}}$, and $C_{\tilde{G}}$ are included simultaneously or individually. It is also worth noting that the bounds on $C_{\tilde{G}}$ and $C_{a \Phi}^{(2)}$ are unchanged compared to the individual limits (16) and (17). Considering that $C_{\tilde{W}}$ is always dominantly constrained by Eq. (19), we conclude that only the unitarity constraint on $C_{\tilde{B}}$ depends significantly on whether $C_{a \Phi}^{(2)}$ is included or not.

\section{Truncating at dimension 5}

Finally, it can be interesting to investigate bounds on the dimension-5 interactions only. As we have seen above, the most stringent bounds on $C_{\tilde{W}}$ originate from processes exhibiting just one dimension-5 vertex; therefore, it is not modified when we truncate the EFT expansion to $\mathcal{O}\left(f_{a}^{-1}\right)$.

To obtain limits on $C_{\tilde{B}}$ and $C_{\tilde{G}}$ independently of assumptions about $C_{a \Phi}^{(2)}$, we can restrict our analysis to a subspace of initial states such that contributions of the dimension- 6 operator are negligible for all the scattering amplitudes retained. This is achieved by eliminating "flavor" states in Eqs. (11) and (12) that lead to processes containing two ALP external legs. We can rederive the constraints on this flavor subspace, and we obtain that the largest eigenvalues come from the $Q=0, T^{0}$ matrix, and they coincide with those in Eq. (20), leading to the bounds in Eqs. (21)-(23). The result is the same, irrespective of whether $C_{\tilde{B}}$ and $C_{\tilde{G}}$ are included simultaneously or individually.

\section{B. Helicity amplitudes involving fermions}

ALP couplings to fermions can contribute to processes

$$
f_{1 \sigma_{1}} \bar{f}_{2 \sigma_{2}} \rightarrow V_{3 \lambda_{3}} V_{4 \lambda_{4}},
$$

which can also violate unitarity. In this case, the partialwave expansion is given by

$$
\begin{aligned}
& \mathcal{M}\left(f_{1 \sigma_{1}} \bar{f}_{2 \sigma_{2}} \rightarrow V_{3 \lambda_{3}} V_{4 \lambda_{4}}\right) \\
& =16 \pi \sum_{J}(2 J+1) \delta_{\sigma_{1}, \sigma_{2}} d_{\sigma_{1}-\sigma_{2}, \lambda_{3}-\lambda_{4}}^{J}(\theta) \\
& \quad \times T^{J}\left(f_{1 \sigma_{1}} \bar{f}_{2 \sigma_{2}} \rightarrow V_{3 \lambda_{3}} V_{4 \lambda_{4}}\right) .
\end{aligned}
$$

In principle, $f_{1 \sigma_{1}} \bar{f}_{2 \sigma_{2}} \rightarrow V_{3 \lambda_{3}} V_{4 \lambda_{4}}$ amplitudes of a given $J$ partial wave can be incorporated together with the $V_{1 \lambda_{1}} V_{2 \lambda_{2}} \rightarrow V_{3 \lambda_{3}} V_{4 \lambda_{4}}$ amplitudes in the corresponding $T^{J}$ matrix by extending the basis of states to incorporate the relevant $f_{1 \sigma_{1}} \bar{f}_{2 \sigma_{2}}$ combinations contributing to a given $Q$; see, for example, Ref. [50]. However, we find that the most energy divergent amplitudes for fermion-antifermion scattering grow at most as $\sqrt{S}$ and, therefore, the contributions from the ALP-fermion couplings enter with different power of $S$ with respect to the ALP-gauge boson couplings in the 
eigenvalues of this generalized $T^{J}$ matrices. Thus, to derive independent unitarity constraints on the $C_{f \Phi}$ couplings, we find it more convenient to follow the alternative procedure presented in Ref. [57] and relate the corresponding $f_{1 \sigma_{1}} \bar{f}_{2 \sigma_{2}} \rightarrow V_{3 \lambda_{3}} V_{4 \lambda_{4}}$ amplitude to that of the elastic process

$$
f_{1 \sigma_{1}} \bar{f}_{2 \sigma_{2}} \rightarrow f_{1 \sigma_{1}} \bar{f}_{2 \sigma_{2}} .
$$

In this case, the unitarity relation is

$$
\begin{aligned}
\operatorname{Im}[ & \left.T^{J}\left(f_{1 \sigma_{1}} \bar{f}_{2 \sigma_{2}} \rightarrow f_{1 \sigma_{1}} \bar{f}_{2 \sigma_{2}}\right)\right] \\
= & \left|T^{J}\left(f_{1 \sigma_{1}} \bar{f}_{2 \sigma_{2}} \rightarrow f_{1 \sigma_{1}} \bar{f}_{2 \sigma_{2}}\right)\right|^{2} \\
& +\sum_{V_{3 \lambda_{3}, V_{4 \lambda_{4}}}\left|T^{J}\left(f_{1 \sigma_{1}} \bar{f}_{2 \sigma_{2}} \rightarrow V_{3 \lambda_{3}} V_{4 \lambda_{4}}\right)\right|^{2}} \\
& +\sum_{N}\left|T^{J}\left(f_{1 \sigma_{1}} \bar{f}_{2 \sigma_{2}} \rightarrow N\right)\right|^{2},
\end{aligned}
$$

where we take the limit $S \gg\left(M_{V_{3}}+M_{V_{4}}\right)^{2},\left(M_{f_{1}}+M_{f_{2}}\right)^{2}$. $N$ represents any state which $f_{1 \sigma_{1}} \bar{f}_{2 \sigma_{2}}$ can annihilate into that does not consist of two bosons. Equation (28) is a quadratic equation for $\operatorname{Im}\left[T^{J}\left(f_{1 \sigma_{1}} \bar{f}_{2 \sigma_{2}} \rightarrow f_{1 \sigma_{1}} \bar{f}_{2 \sigma_{2}}\right)\right]$, which only admits a solution if

$$
\sum_{V_{3 \lambda_{3},}, V_{4 \lambda_{4}}}\left|T^{J}\left(f_{1 \sigma_{1}} \bar{f}_{2 \sigma_{2}} \rightarrow V_{3 \lambda_{3}} V_{4 \lambda_{4}}\right)\right|^{2} \leq \frac{1}{4}
$$

The strongest bounds can be found by considering some optimized linear combinations

$$
|X\rangle=\sum_{f_{1}, \sigma_{1}} x_{f_{2}, \sigma_{2}}\left|f_{1 \sigma_{1}} \bar{f}_{2 \sigma_{2}}\right\rangle
$$

with the normalization condition $\sum_{f \sigma}\left|x_{f \sigma}\right|^{2}=1$, for which the amplitude $T^{J}\left(X \rightarrow V_{3 \lambda_{3}} V_{4 \lambda_{4}}\right)$ is the largest.

In this approach, processes of fermion scattering into one gauge boson and one ALP provide independent constraints on the ALP-fermion coupling. As mentioned above, the most divergent relevant helicity amplitudes grow as $\sqrt{S}$ and are listed in Table V. For couplings to leptons of a given generation, the strongest bounds are obtained with $|X\rangle=$ $\frac{1}{\sqrt{2}}\left|e_{+}^{-} e_{+}^{+}+e_{-}^{-} e_{-}^{+}\right\rangle$(or equivalently with $|X\rangle=\left|\nu_{-} e_{-}^{+}\right\rangle$). For couplings to quarks of a given generation, accounting for the $N_{C}=3$ color states, the strongest bounds are obtained with $|X\rangle=\frac{1}{\sqrt{2 N_{c}}} \sum_{a=1}^{N_{c}}\left|q_{+}^{a} \bar{q}_{+}^{a}+q_{-}^{a} \bar{q}_{-}^{a}\right\rangle$, or equivalently with $\frac{1}{\sqrt{N_{c}}} \sum_{a=1}^{N_{c}}\left|u_{+}^{a} \bar{d}_{+}^{a}\right\rangle$ and $\left.\frac{1}{\sqrt{N_{c}}} \sum_{a=1}^{N_{c}}\left|u_{-}^{a} \bar{d}_{-}^{a}\right\rangle\right)$. Furthermore, within the assumed flavour symmetry of the axion coupling to fermions, the strongest bounds correspond to the processes with fermions of the third generation, and they read

$$
\begin{array}{r}
\left|C_{a \Phi}-C_{e \Phi}\right| \leq \frac{16 \pi}{\left|Y_{\tau}\right|}\left(\frac{f_{a}}{\sqrt{S}}\right)=\frac{50}{\left|Y_{\tau}\right|} \frac{f_{a}}{\mathrm{TeV}}\left(\frac{\mathrm{TeV}}{\sqrt{S}}\right), \\
\left|C_{a \Phi}+C_{u \Phi}\right| \leq \frac{16 \pi}{\sqrt{N_{C}}\left|Y_{t}\right|}\left(\frac{f_{a}}{\sqrt{S}}\right)=\frac{29}{\left|Y_{t}\right|} \frac{f_{a}}{\mathrm{TeV}}\left(\frac{\mathrm{TeV}}{\sqrt{S}}\right), \\
\left|C_{a \Phi}-C_{d \Phi}\right| \leq \frac{16 \pi}{\sqrt{N_{C}}\left|Y_{b}\right|}\left(\frac{f_{a}}{\sqrt{S}}\right)=\frac{29}{\left|Y_{b}\right|} \frac{f_{a}}{\mathrm{TeV}}\left(\frac{\mathrm{TeV}}{\sqrt{S}}\right) .
\end{array}
$$

Because these bounds are inversely proportional to the Yukawa coupling of the fermion and involve larger coefficients, we conclude that the unitarity constraints on the ALP-fermion couplings are orders of magnitude weaker than those on ALP-gauge boson couplings even for the coupling to the up quarks. Moreover, the operator $\mathcal{O}_{a \Phi}$ should only be considered in a scenario where the fermionic operators are absent. In this case, the most stringent unitarity constraint on its coupling originates from Eq. (32).

\section{CONCLUSIONS}

We have derived maximal constraints on the effective interactions of axionlike particles from partial-wave unitarity in $2 \rightarrow 2$ scattering processes. Our results are summarized in Fig. 1. They hold in the kinematic regime where $\sqrt{S} \gg v$, and the ALP mass was also implicitly

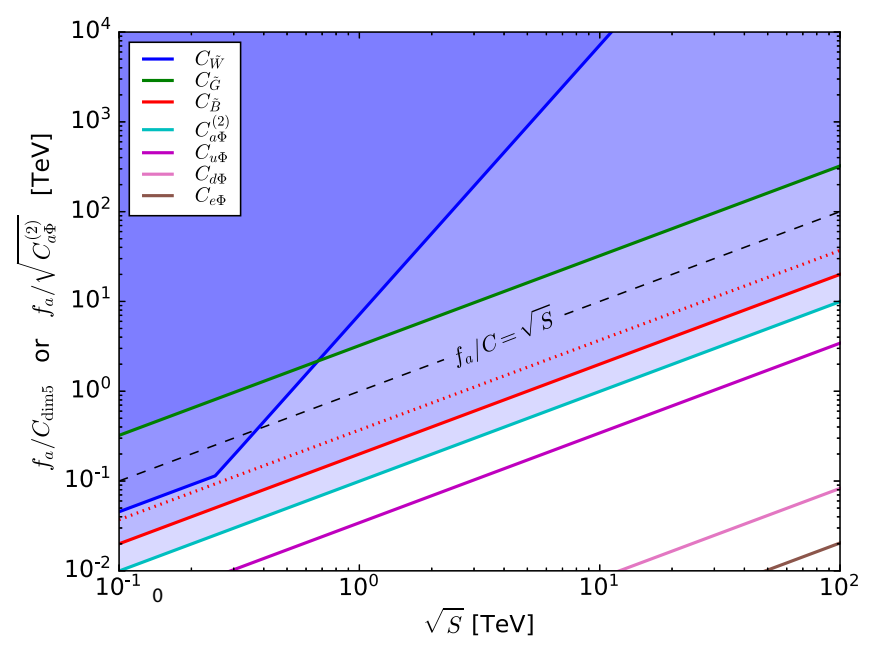

FIG. 1. Summary of unitarity bounds derived in this work. The shaded regions indicate allowed values of $f_{a} / C\left(f_{a} / \sqrt{C}\right.$ in the case of $C_{a \Phi}^{(2)}$ ) and $\sqrt{S}$ for each bosonic interaction. For $C_{\tilde{W}}$, we plot the most stringent bound between Eq. (14) and (19). The solid red line indicates the bound on $C_{\tilde{B}}$ in Eq. (22), derived allowing $C_{a \Phi}^{(2)}$ to vary simultaneously. The individual bound on $C_{\tilde{B}}$ given in Eq. (15) is drawn as a dotted line in the same color. The bounds on fermionic operators are always subdominant and are only marked as solid lines. The bound on $C_{a \Phi}$, that should only considered in a setup where the fermionic operators are absent, coincides with that on $C_{u \Phi}$. Finally, the gray dashed line marks the diagonal for reference. 
taken to be $m_{a} \ll \sqrt{S}$. Furthermore, the consistency of the ALP EFT expansion requires $\sqrt{S} \ll f_{a}$.

We find that, for fixed $C / f_{a}$, the most stringent unitarity bound is imposed on $C_{\tilde{W}}$ in $V V \rightarrow V a$ scattering processes, while the weakest limits are on ALP-fermion interactions. The constraints exhibit only a limited dependence on whether the effective operators are taken individually or allowed to vary simultaneously, signaling that each of them is dominantly constrained in a class of scattering amplitudes that is nearly orthogonal to the others.

The constraints we have derived can be particularly relevant for ALP searches at colliders [65,74,75,79-91], where, depending on their masses, ALP particles could be observed in $a \rightarrow X X$ decays, with $X$ a generic SM state, in $X a$ associated production (with the ALP either going undetected or decaying to photons or light fermions) or in nonresonant $2 \rightarrow 2$ scattering processes, where the ALP appears as an off-shell internal line [86,90,91].

In this respect, let us stress that our results should not be interpreted as strict unitarity constraints on any specific process used in the ALP searches, in the sense that it might be difficult to directly identify the kinematic information available with the subprocess center-of-mass energy of an individual $2 \rightarrow 2$ scattering. Notwithstanding, unitarity bounds must be satisfied in the event generation and, consequently, can affect the shapes of expected distributions used in the searches.

For example, recently, the ATLAS Collaboration has searched for axions in events with an energetic jet [92] or a photon [93] and missing transverse momentum. The monojet analysis [92] constrains the axion coupling to gluons to satisfy $C_{\tilde{G}} / f_{a}<0.008 \mathrm{TeV}^{-1}$ at $95 \%$ C.L. Using Eq. (23), we find that for the largest allowed coupling in this search unitarity is preserved up to center mass-of-mass energy of $39 \mathrm{TeV}$, clearly beyond the LHC reach. On the other hand, the monophoton analysis limits the $C_{\tilde{W}}$ coupling to satisfy $C_{\tilde{W}} / f_{a}<0.12 \mathrm{TeV}^{-1}$ at $95 \%$ C.L. From Eq. (19), we read that for $C_{\tilde{W}} / f_{a}$ at the $95 \%$ C.L. boundary unitarity is violated in subprocesses with center-of-mass energy greater than $1.04 \mathrm{TeV}$. We conclude that the tail of the expected missing $E_{T}$ distribution should be analyzed cautiously and the unitarity constraints could have an impact in the derivation of the monophoton search bound.

The unitarity bounds derived in this work would be also relevant in the event that an ALP signal will be detected in the future (independently of the energy regime at which the experimental search is conducted), leading to a defined measurement of one or more ALP couplings. In this case, unitarity bounds would provide an upper limit to the mass scale of the new physics sector the ALP originates from and motivate further searches in this energy region.

\section{ACKNOWLEDGMENTS}

O. J. P. E. is supported in part by Conselho Nacional de Desenvolvimento Centífico e Tecnológico (CNPq) and by Fundação de Amparo à Pesquisa do Estado de São Paulo (FAPESP) Grant No. 2019/04837-9. M. C. G.-G. is supported by Spanish Grant No. PID2019-105614 GB-C21, by U.S. NSF Grant No. PHY-1915093, and by AGAUR (Generalitat de Catalunya) Grant No. 2017-SGR-929. The authors acknowledge the support of European ITN Grant No. H2020-MSCA-ITN-2019//860881-HIDDeN.

\section{APPENDIX: HELICITY AMPLITUDES AT LEADING ORDER IN $S$}

We present here the list of unitarity violating amplitudes for all the $2 \rightarrow 2$ scattering processes considered in the evaluation of the unitarity constraints.

TABLE I. Leading contributions to the helicity amplitudes for the channels involving SM bosons and even number of gluons in the initial or final state and with projections in $J=0$. They contribute to the $T^{J}$ matrices with $Q=2,1,0$ and $J=0,1$. In the expressions, $\mathrm{X}_{+}=(1+\cos x)$ and $\mathrm{X}_{-}=(1-\cos x)$, where $x$ is the polar angle.

\begin{tabular}{|c|c|c|c|c|c|}
\hline & $\lambda_{1}$ & $\lambda_{2}$ & $\lambda_{3}$ & $\lambda_{4}$ & $\mathcal{M}\left(\times \frac{S}{f_{a}^{2}}\right)$ \\
\hline$W^{+} W^{+} \rightarrow W^{+} W^{+}$ & $\begin{array}{c}1 \\
-1\end{array}$ & $\begin{array}{c}-1 \\
1\end{array}$ & $\begin{array}{c}1 \\
\mp 1\end{array}$ & $\begin{array}{c}1 \\
\pm 1\end{array}$ & $\begin{array}{c}2 C_{\tilde{W}}^{2} \\
X_{ \pm} C_{\tilde{W}}^{2}\end{array}$ \\
\hline $\begin{array}{l}W^{+} Z \rightarrow W^{+} Z \\
W^{+} Z \rightarrow W^{+} \gamma \\
W^{+} \gamma \rightarrow W^{+} \gamma\end{array}$ & $\begin{array}{l}-1 \\
-1 \\
-1\end{array}$ & $\begin{array}{l}\mp 1 \\
\mp 1 \\
\mp 1\end{array}$ & $\begin{array}{l}1 \\
1 \\
1\end{array}$ & $\begin{array}{l} \pm 1 \\
\pm 1 \\
\pm 1\end{array}$ & $\begin{array}{l} \pm 2 C_{\tilde{W}} X_{-}\left(s_{w}^{2} C_{\tilde{B}}+c_{w}^{2} C_{\tilde{W}}\right) \\
\mp 2 c_{w} C_{\tilde{W}} s_{w} X_{-}\left(C_{\tilde{B}}-C_{\tilde{W}}\right) \\
\pm 2 C_{\tilde{W}} X_{-}\left(c_{w}^{2} C_{\tilde{B}}+s_{w}^{2} C_{\tilde{W}}\right)\end{array}$ \\
\hline$W^{+} W^{-} \rightarrow W^{+} W^{-}$ & $\begin{array}{l}-1 \\
-1\end{array}$ & $\begin{array}{l}-1 \\
\mp 1\end{array}$ & $\begin{array}{c}-1 \\
1\end{array}$ & $\begin{array}{l}-1 \\
\pm 1\end{array}$ & $\begin{array}{c}4 C_{\tilde{\tilde{W}}}^{2} \\
-2 C_{\tilde{W}}^{2} X_{ \pm}\end{array}$ \\
\hline $\begin{array}{l}W^{+} W^{-} \rightarrow Z Z \\
W^{+} W^{-} \rightarrow Z \gamma \\
W^{+} W^{-} \rightarrow \gamma \gamma\end{array}$ & $\begin{array}{l}-1 \\
-1 \\
-1\end{array}$ & $\begin{array}{l}-1 \\
-1 \\
-1\end{array}$ & $\begin{array}{l}\mp 1 \\
\pm 1 \\
\mp 1\end{array}$ & $\begin{array}{l}\mp 1 \\
\pm 1 \\
\mp 1\end{array}$ & $\begin{array}{l} \pm 2 \sqrt{2} C_{\tilde{W}}\left(s_{w}^{2} C_{\tilde{B}}+c_{w}^{2} C_{\tilde{W}}\right) \\
\quad \pm 4 c_{w} C_{\tilde{W}} s_{w}\left(C_{\tilde{B}}-C_{\tilde{W}}\right) \\
\pm 2 \sqrt{2} C_{\tilde{W}}\left(c_{w}^{2} C_{\tilde{B}}+s_{w}^{2} C_{\tilde{W}}\right)\end{array}$ \\
\hline
\end{tabular}


TABLE I. (Continued)

\begin{tabular}{|c|c|c|c|c|c|}
\hline & $\lambda_{1}$ & $\lambda_{2}$ & $\lambda_{3}$ & $\lambda_{4}$ & $\mathcal{M}\left(\times \frac{S}{f_{a}^{2}}\right)$ \\
\hline$Z Z \rightarrow Z Z$ & $\begin{array}{l}-1 \\
-1\end{array}$ & $\begin{array}{c}-1 \\
1\end{array}$ & $\begin{array}{l}-1 \\
\mp 1\end{array}$ & $\begin{array}{l}-1 \\
\pm 1\end{array}$ & $\begin{aligned} & 2\left(c_{w}^{2} C_{\tilde{W}}-s_{w}^{2} C_{\tilde{B}}\right)^{2} \\
- & X_{ \pm}\left(c_{w}^{2} C_{\tilde{W}}-s_{w}^{2} C_{\tilde{B}}\right)^{2}\end{aligned}$ \\
\hline$Z Z \rightarrow Z \gamma$ & $\begin{array}{l}-1 \\
-1\end{array}$ & $\begin{array}{l}-1 \\
-1\end{array}$ & $\begin{array}{l}-1 \\
\mp 1\end{array}$ & $\begin{array}{l}-1 \\
\pm 1\end{array}$ & $\begin{array}{c}2 \sqrt{2} c_{w} s_{w}\left(C_{\tilde{B}}-C_{\tilde{W}}\right)\left(s_{w}^{2} C_{\tilde{B}}-c_{w}^{2} C_{\tilde{W}}\right) \\
-\sqrt{2} X_{ \pm} c_{w} s_{w}\left(C_{\tilde{B}}-C_{\tilde{W}}\right)\left(s_{w}^{2} C_{\tilde{B}}-c_{w}^{2} C_{\tilde{W}}\right)\end{array}$ \\
\hline$Z Z \rightarrow \gamma \gamma$ & $\begin{array}{l}-1 \\
-1 \\
-1\end{array}$ & $\begin{array}{c}-1 \\
-1 \\
1\end{array}$ & $\begin{array}{c}-1 \\
1 \\
\mp 1\end{array}$ & $\begin{array}{l}-1 \\
1 \\
\pm 1\end{array}$ & $\begin{array}{c}-2\left(\left(C_{\tilde{B}}^{2}+C_{\tilde{W}}^{2}\right) c_{w}^{2} s_{w}^{2}-C_{\tilde{B}} C_{\tilde{W}}\left(c_{w}^{4}+s_{w}^{4}\right)\right) \\
-2 C_{\tilde{B}} C_{\tilde{W}} \\
\mathrm{X}_{ \pm}\left(C_{\tilde{B}}-C_{\tilde{W}}\right)^{2} c_{w}^{2} s_{w}^{2}\end{array}$ \\
\hline$Z \gamma \rightarrow Z \gamma$ & $\begin{array}{l}-1 \\
-1 \\
-1\end{array}$ & $\begin{array}{c}-1 \\
-1 \\
1\end{array}$ & $\begin{array}{c}-1 \\
1 \\
\mp 1\end{array}$ & $\begin{array}{l}-1 \\
1 \\
\pm 1\end{array}$ & $\begin{array}{c}4 c_{w}^{2} s_{w}^{2}\left(C_{\tilde{B}}-C_{\tilde{W}}\right)^{2} \\
-2 C_{\tilde{B}} C_{\tilde{W}} X_{-} \\
-2 X_{ \pm}\left(C_{\tilde{B}}-C_{\tilde{W}}\right)^{2} c_{w}^{2} s_{w}^{2}\end{array}$ \\
\hline$\gamma \gamma \rightarrow \gamma \gamma$ & $\begin{array}{l}-1 \\
-1\end{array}$ & $\begin{array}{c}-1 \\
1\end{array}$ & $\begin{array}{l}-1 \\
\mp 1\end{array}$ & $\begin{array}{l}-1 \\
\pm 1\end{array}$ & $\begin{array}{c}2\left(c_{w}^{2} C_{\tilde{B}}+s_{w}^{2} C_{\tilde{W}}\right)^{2} \\
-X_{ \pm}\left(c_{w}^{2} C_{\tilde{B}}+s_{w}^{2} C_{\tilde{W}}\right)^{2}\end{array}$ \\
\hline $\begin{array}{l}W^{+} W^{-} \rightarrow G^{a} G^{b} \\
Z Z \rightarrow G^{a} G^{b} \\
Z \gamma \rightarrow G^{a} G^{b} \\
\gamma \gamma \rightarrow G^{a} G^{b}\end{array}$ & $\begin{array}{l}-1 \\
-1 \\
-1 \\
-1\end{array}$ & $\begin{array}{l}-1 \\
-1 \\
-1 \\
-1\end{array}$ & $\begin{array}{l}\mp 1 \\
\mp 1 \\
\mp 1 \\
\mp 1\end{array}$ & $\begin{array}{l}\mp 1 \\
\mp 1 \\
\mp 1 \\
\mp 1\end{array}$ & $\begin{array}{c} \pm 8 \sqrt{2} C_{\tilde{G}} C_{\tilde{W}}, \delta_{a b} \\
\pm 8 C_{\tilde{G}}\left(s_{w}^{2} C_{\tilde{B}}+c_{w}^{2} C_{\tilde{W}}\right) \delta_{a b} \\
\mp 8 \sqrt{2} C_{\tilde{G}} c_{w} s_{w}\left(C_{\tilde{B}}-C_{\tilde{W}}\right) \delta_{a b} \\
\pm 8 C_{\tilde{G}}\left(s_{w}^{2} C_{\tilde{B}}+c_{w}^{2} C_{\tilde{W}}\right) \delta_{a b}\end{array}$ \\
\hline$G^{a} G^{b} \rightarrow G^{c} G^{d}$ & $\begin{array}{l}-1 \\
-1 \\
-1 \\
-1\end{array}$ & $\begin{array}{c}-1 \\
-1 \\
1 \\
1\end{array}$ & $\begin{array}{c}-1 \\
1 \\
-1 \\
1\end{array}$ & $\begin{array}{c}-1 \\
1 \\
1 \\
-1\end{array}$ & $\begin{array}{c}32 C_{\tilde{G}}^{2} \delta_{a b} \delta_{c d} \\
16 C_{\tilde{G}}^{2}\left(X_{-} \delta_{a c} \delta_{b d}+X_{+} \delta_{a d} \delta_{b c}-2 \delta_{a b} \delta_{c d}\right) \\
-4 X_{+} C_{\tilde{G}}^{2} \delta_{a c} \delta_{b d} \\
-4 X_{-} C_{\tilde{G}}^{2} \delta_{a d} \delta_{b c}\end{array}$ \\
\hline
\end{tabular}

TABLE II. Leading contributions to the helicity amplitudes for the channels involving SM bosons and two ALPs and an even number of gluons in the initial or final state. They contribute to the $T^{J}$ matrices with $Q=1,0$ and $J=0$ or $J=1$. In the expressions, $\mathrm{X}_{+}=(1+\cos x)$ and $\mathrm{X}_{-}=(1-\cos x)$, where $x$ is the polar angle.

\begin{tabular}{|c|c|c|c|c|c|}
\hline & $\lambda_{1}$ & $\lambda_{2}$ & $\lambda_{3}$ & $\lambda_{4}$ & $\mathcal{M}\left(\times \frac{S}{f_{a}^{2}}\right)$ \\
\hline \multirow[t]{3}{*}{$W^{+} a \rightarrow W^{+} a$} & -1 & 0 & -1 & 0 & $-2 C_{\tilde{W}}^{2} \mathrm{X}_{-}$ \\
\hline & -1 & 0 & 1 & 0 & $4 C_{\tilde{W}}^{2} \mathrm{X}_{-}$ \\
\hline & 0 & 0 & 0 & 0 & $\frac{1}{2} C_{a \Phi}^{(2)} \mathrm{X}_{-}$ \\
\hline \multirow[t]{3}{*}{$W^{+} W^{-} \rightarrow a a$} & -1 & -1 & 0 & 0 & $4 \sqrt{2} C_{\tilde{W}}^{2}$ \\
\hline & -1 & 1 & 0 & 0 & $-2 \sqrt{2} C_{\tilde{W}}^{2}$ \\
\hline & 0 & 0 & 0 & 0 & $\frac{1}{\sqrt{2}} C_{a \Phi}^{(2)}$ \\
\hline \multirow[t]{3}{*}{$Z Z \rightarrow a a$} & -1 & -1 & 0 & 0 & $4\left(s_{w}^{2} C_{\tilde{B}}^{2}+c_{w}^{2} C_{\tilde{W}}^{2}\right)$ \\
\hline & -1 & 1 & 0 & 0 & $-2\left(s_{w}^{2} C_{\tilde{B}}^{D}+c_{w}^{2} C_{\tilde{W}}^{2}\right)$ \\
\hline & 0 & 0 & 0 & 0 & $C_{a \Phi}^{(2)}$ \\
\hline \multirow[t]{2}{*}{$Z \gamma \rightarrow a a$} & -1 & -1 & 0 & 0 & $-4 \sqrt{2} c_{w} s_{w}\left(C_{\tilde{B}}^{2}-C_{\tilde{W}}^{2}\right)$ \\
\hline & -1 & 1 & 0 & 0 & $2 \sqrt{2} c_{w} s_{w}\left(C_{\tilde{B}}^{2}-C_{\tilde{W}}^{2}\right)$ \\
\hline \multirow[t]{3}{*}{$Z a \rightarrow Z a$} & -1 & 0 & -1 & 0 & $-2 \mathrm{X}_{-}\left(s_{w}^{2} C_{\tilde{B}}^{2}+c_{w}^{2} C_{\tilde{W}}^{2}\right)$ \\
\hline & -1 & 0 & 1 & 0 & $4 \mathrm{X}_{-}\left(s_{w}^{2} C_{\tilde{B}}^{2}+c_{w}^{2} C_{\tilde{W}}^{2}\right)$ \\
\hline & 0 & 0 & 0 & 0 & $\frac{1}{2} C_{a \Phi}^{(2)} \mathrm{X}_{-}$ \\
\hline \multirow[t]{2}{*}{$Z a \rightarrow \gamma a$} & -1 & 0 & -1 & 0 & $2 c_{w} s_{w} \mathrm{X}_{-}\left(C_{\tilde{B}}^{2}-C_{\tilde{W}}^{2}\right)$ \\
\hline & -1 & 0 & 1 & 0 & $-4 c_{w} s_{w} \mathrm{X}_{-}\left(C_{\tilde{B}}^{2}-C_{\tilde{W}}^{2}\right)$ \\
\hline
\end{tabular}


TABLE II. (Continued)

\begin{tabular}{|c|c|c|c|c|c|}
\hline & $\lambda_{1}$ & $\lambda_{2}$ & $\lambda_{3}$ & $\lambda_{4}$ & $\mathcal{M}\left(\times \frac{S}{f_{a}^{2}}\right)$ \\
\hline \multirow[t]{2}{*}{$\gamma \gamma \rightarrow a a$} & -1 & -1 & 0 & 0 & $4\left(c_{w}^{2} C_{\tilde{B}}^{2}+s_{w}^{2} C_{\tilde{W}}^{2}\right)$ \\
\hline & -1 & 1 & 0 & 0 & $-2\left(c_{w}^{2} C_{\tilde{B}}^{B}+s_{w}^{2} C_{\tilde{W}}^{2}\right)$ \\
\hline \multirow[t]{2}{*}{$\gamma a \rightarrow \gamma a$} & -1 & 0 & -1 & 0 & $-2 \mathrm{X}_{-}\left(c_{w}^{2} C_{\tilde{B}}^{2}+s_{w}^{2} C_{\tilde{W}}^{2}\right)$ \\
\hline & -1 & 0 & 1 & 0 & $4 \mathrm{X}_{-}\left(c_{w}^{2} C_{\tilde{B}}^{2}+s_{w}^{2} C_{\tilde{W}}^{2}\right)$ \\
\hline \multirow[t]{2}{*}{$G^{a} G^{b} \rightarrow a a$} & -1 & -1 & 0 & 0 & $16 C_{\tilde{G}}^{2} \delta_{a b}$ \\
\hline & -1 & 11 & 0 & 0 & $-8 C_{\tilde{G}}^{2} \delta_{a b}$ \\
\hline$H a \rightarrow H a$ & 0 & 0 & 0 & 0 & $\frac{1}{2} C_{a \Phi}^{(2)} \mathrm{X}_{-}$ \\
\hline$H H \rightarrow a a$ & 0 & 0 & 0 & 0 & $\frac{1}{2} C_{a \Phi}^{(2)}$ \\
\hline
\end{tabular}

TABLE III. Leading contributions to the helicity amplitudes for the channels with one gluon in the initial and final states. They contribute $\bar{T}^{J}$ with $Q=1,0$ and $J=0,1 . \mathrm{X}_{+}=(1+\cos x)$ and $\mathrm{X}_{-}=(1-\cos x)$, where $x$ is the polar angle.

\begin{tabular}{|c|c|c|c|c|c|}
\hline & $\lambda_{1}$ & $\lambda_{2}$ & $\lambda_{3}$ & $\lambda_{4}$ & $\mathcal{M}\left(\times \frac{S}{f_{a}^{2}}\right)$ \\
\hline$W^{+} G^{a} \rightarrow W^{+} G^{b}$ & -1 & $\mp 1$ & 1 & \pm 1 & $\pm 8 C_{\tilde{G}} C_{\tilde{W}} \mathrm{X}_{-} \delta_{a b}$ \\
\hline$Z G^{a} \rightarrow Z G^{b}$ & -1 & $\mp 1$ & 1 & \pm 1 & $\pm 8 C_{\tilde{G}} \mathrm{X}_{-}\left(s_{w}^{2} C_{\tilde{B}}+c_{w}^{2} C_{\tilde{W}}\right) \delta_{a b}$ \\
\hline$Z G^{a} \rightarrow \gamma G^{b}$ & -1 & $\mp 1$ & 1 & \pm 1 & $\mp 8 C_{\tilde{G}} c_{w} s_{w} \mathrm{X}_{-}\left(C_{\tilde{B}}-C_{\tilde{W}}\right) \delta_{a b}$ \\
\hline$\gamma G^{a} \rightarrow \gamma G^{b}$ & -1 & $\mp 1$ & 1 & \pm 1 & $\pm 8 C_{\tilde{G}} \mathrm{X}_{-}\left(c_{w}^{2} C_{\tilde{B}}+s_{w}^{2} C_{\tilde{W}}\right) \delta_{a b}$ \\
\hline \multirow[t]{2}{*}{$G^{a} a \rightarrow G^{b} a$} & -1 & 0 & -1 & 0 & $-8 C_{\tilde{G}}^{2} \mathrm{X}_{-} \delta_{a b}$ \\
\hline & -1 & 0 & 1 & 0 & $16 C_{\tilde{G}}^{2} \mathrm{X}_{-} \delta_{a b}$ \\
\hline
\end{tabular}

TABLE IV. Leading contributions to the helicity amplitudes for the channels involving SM bosons and one ALP. They contribute to the $T^{J}$ matrices with $Q=1,0$ and $J=1$. In these amplitudes, $\mathrm{Y}=\sin x$, where $x$ is the polar angle.

\begin{tabular}{|c|c|c|c|c|c|}
\hline & $\lambda_{1}$ & $\lambda_{2}$ & $\lambda_{3}$ & $\lambda_{4}$ & $\mathcal{M}\left(\times e \frac{S^{3 / 2}}{f_{a} M_{W}^{2}}\right)$ \\
\hline \multirow[t]{3}{*}{$W^{+} W^{-} \rightarrow Z a$} & -1 & 0 & 0 & 0 & $\sqrt{2} \frac{c_{w}^{2}}{s_{w}} C_{\tilde{W}} \mathrm{Y}$ \\
\hline & 0 & -1 & 0 & 0 & $-\sqrt{2} \frac{c_{w}^{2}}{s_{w}} C_{\tilde{W}} \mathrm{Y}$ \\
\hline & 0 & 0 & -1 & 0 & $\sqrt{2} \frac{c_{w}^{w}}{s_{w}} C_{\tilde{W}} \mathrm{Y}$ \\
\hline$W^{+} W^{-} \rightarrow \gamma a$ & 0 & 0 & -1 & 0 & $\sqrt{2} C_{\tilde{W}} \mathrm{Y}$ \\
\hline \multirow[t]{3}{*}{$W^{+} Z \rightarrow W^{+} a$} & -1 & 0 & 0 & 0 & $-\sqrt{2} \frac{c_{w}^{2}}{s_{w}} C_{\tilde{W}} \mathrm{Y}$ \\
\hline & 0 & -1 & 0 & 0 & $\sqrt{2} \frac{c_{w}^{2}}{s_{w}} C_{\tilde{W}} \mathrm{Y}$ \\
\hline & 0 & 0 & -1 & 0 & $-\sqrt{2} \frac{c_{w}^{2}}{s_{w}} C_{\tilde{W}} \mathrm{Y}$ \\
\hline$W^{+} \gamma \rightarrow W^{+} a$ & 0 & -1 & 0 & 0 & $\sqrt{2} C_{\tilde{W}} \mathrm{Y}$ \\
\hline
\end{tabular}


TABLE V. Leading contributions to the helicity amplitudes for the channels with fermion scattering. $a, b$ denote color indices, and $r, s$ denote flavor indices.

\begin{tabular}{lccccc}
\hline \hline & $\sigma_{1}$ & $\sigma_{2}$ & $\lambda_{3}$ & $\lambda_{4}$ & $\mathcal{M}\left(i \frac{\sqrt{S}}{f_{a}}\right)$ \\
\hline$e_{r}^{-} e_{s}^{+} \rightarrow Z a$ & - & - & 0 & 0 & $\frac{1}{\sqrt{2}}\left(C_{a \Phi}-C_{e \Phi}\right)\left(Y_{e}^{*}\right)_{r s}$ \\
& + & + & 0 & 0 & $\frac{1}{\sqrt{2}}\left(C_{a \Phi}-C_{e \Phi}\right)\left(Y_{e}\right)_{s r}$ \\
$\nu_{r} e_{s}^{+} \rightarrow W^{+} a$ & - & - & 0 & 0 & $\left(C_{a \Phi}-C_{e \Phi}\right)\left(Y_{e}^{*}\right)_{r s}$ \\
$u_{r}^{a} \bar{u}_{s}^{b} \rightarrow Z a$ & - & - & 0 & 0 & $\frac{1}{\sqrt{2}}\left(C_{a \Phi}+C_{u \Phi}\right)\left(Y_{u}^{*}\right)_{r s} \delta_{a b}$ \\
& + & + & 0 & 0 & $\frac{1}{\sqrt{2}}\left(C_{a \Phi}+C_{u \Phi}\right)\left(Y_{u}\right)_{s r} \delta_{a b}$ \\
$d_{r}^{a} \bar{d}_{s}^{b} \rightarrow Z a$ & - & - & 0 & 0 & $\frac{1}{\sqrt{2}}\left(C_{a \Phi}-C_{d \Phi}\right)\left(Y_{d}^{*}\right)_{r s} \delta_{a b}$ \\
& + & - & 0 & 0 & $\frac{1}{\sqrt{2}}\left(C_{a \Phi}-C_{d \Phi}\right)\left(Y_{d}\right)_{s r} \delta_{a b}$ \\
$u_{r}^{a} \bar{d}_{s}^{b} \rightarrow W^{+} a$ & - & - & 0 & 0 & $\left(C_{a \Phi}-C_{d \Phi}\right)\left(Y_{d}^{*}\right)_{r s} \delta_{a b}$ \\
& + & + & 0 & 0 & $\left(C_{a \Phi}+C_{u \Phi}\right)\left(Y_{u}\right)_{s r} \delta_{a b}$ \\
\hline \hline
\end{tabular}

[1] R. D. Peccei and H. R. Quinn, Phys. Rev. Lett. 38, 1440 (1977).

[2] R. D. Peccei and H. R. Quinn, Phys. Rev. D 16, 1791 (1977).

[3] S. Weinberg, Phys. Rev. Lett. 40, 223 (1978).

[4] F. Wilczek, Phys. Rev. Lett. 40, 279 (1978).

[5] J. E. Kim, Phys. Rev. Lett. 43, 103 (1979).

[6] M. A. Shifman, A. I. Vainshtein, and V. I. Zakharov, Nucl. Phys. B166, 493 (1980).

[7] M. Dine, W. Fischler, and M. Srednicki, Phys. Lett. 104B, 199 (1981).

[8] A. R. Zhitnitsky, Sov. J. Nucl. Phys. 31, 260 (1980), https:// inspirehep.net/literature/157263.

[9] V. A. Rubakov, JETP Lett. 65, 621 (1997).

[10] Z. Berezhiani, L. Gianfagna, and M. Giannotti, Phys. Lett. B 500, 286 (2001).

[11] S. D. H. Hsu and F. Sannino, Phys. Lett. B 605, 369 (2005).

[12] A. Hook, Phys. Rev. Lett. 114, 141801 (2015).

[13] H. Fukuda, K. Harigaya, M. Ibe, and T. T. Yanagida, Phys. Rev. D 92, 015021 (2015).

[14] C.-W. Chiang, H. Fukuda, M. Ibe, and T. T. Yanagida, Phys. Rev. D 93, 095016 (2016).

[15] T. Gherghetta, N. Nagata, and M. Shifman, Phys. Rev. D 93, 115010 (2016).

[16] S. Dimopoulos, A. Hook, J. Huang, and G. MarquesTavares, J. High Energy Phys. 11 (2016) 052.

[17] A. Kobakhidze, arXiv:1607.06552.

[18] P. Agrawal and K. Howe, J. High Energy Phys. 12 (2018) 029.

[19] P. Agrawal and K. Howe, J. High Energy Phys. 12 (2018) 035.

[20] M. K. Gaillard, M. B. Gavela, R. Houtz, P. Quilez, and R. Del Rey, Eur. Phys. J. C 78, 972 (2018).

[21] M. A. Buen-Abad and J. Fan, J. High Energy Phys. 12 (2019) 161.
[22] C. Csáki, M. Ruhdorfer, and Y. Shirman, J. High Energy Phys. 04 (2020) 031.

[23] A. Hook, S. Kumar, Z. Liu, and R. Sundrum, Phys. Rev. Lett. 124, 221801 (2020).

[24] T. Gherghetta and M. D. Nguyen, J. High Energy Phys. 12 (2020) 094.

[25] A. Hook, Phys. Rev. Lett. 120, 261802 (2018).

[26] L. Di Luzio, B. Gavela, P. Quilez, and A. Ringwald, J. High Energy Phys. 05 (2021) 184.

[27] L. Di Luzio, B. Gavela, P. Quilez, and A. Ringwald, arXiv:2102.01082.

[28] B. Gripaios, A. Pomarol, F. Riva, and J. Serra, J. High Energy Phys. 04 (2009) 070.

[29] B. Gripaios, M. Nardecchia, and T. You, Eur. Phys. J. C 77, 28 (2017).

[30] L. Merlo, F. Pobbe, and S. Rigolin, Eur. Phys. J. C 78, 415 (2018); 79, 963(E) (2019).

[31] M. Chala, G. Durieux, C. Grojean, L. de Lima, and O. Matsedonskyi, J. High Energy Phys. 06 (2017) 088.

[32] I. Brivio, M. B. Gavela, S. Pascoli, R. del Rey, and S. Saa, Chin. J. Phys. (Taipei) 61, 55 (2019).

[33] Y. Chikashige, R. N. Mohapatra, and R. D. Peccei, Phys. Lett. 98B, 265 (1981).

[34] G. B. Gelmini and M. Roncadelli, Phys. Lett. 99B, 411 (1981).

[35] A. Davidson and K. C. Wali, Phys. Rev. Lett. 48, 11 (1982).

[36] F. Wilczek, Phys. Rev. Lett. 49, 1549 (1982).

[37] L. Calibbi, F. Goertz, D. Redigolo, R. Ziegler, and J. Zupan, Phys. Rev. D 95, 095009 (2017).

[38] Y. Ema, K. Hamaguchi, T. Moroi, and K. Nakayama, J. High Energy Phys. 01 (2017) 096.

[39] P. Svrcek and E. Witten, J. High Energy Phys. 06 (2006) 051.

[40] A. Arvanitaki, S. Dimopoulos, S. Dubovsky, N. Kaloper, and J. March-Russell, Phys. Rev. D 81, 123530 (2010). 
[41] H. Georgi, D. B. Kaplan, and L. Randall, Phys. Lett. 169B, 73 (1986).

[42] K. Choi, K. Kang, and J. E. Kim, Phys. Lett. B 181, 145 (1986).

[43] K. Choi, S. H. Im, C. B. Park, and S. Yun, J. High Energy Phys. 11 (2017) 070.

[44] M. Chala, G. Guedes, M. Ramos, and J. Santiago, Eur. Phys. J. C 81, 181 (2021).

[45] M. Bauer, M. Neubert, S. Renner, M. Schnubel, and A. Thamm, J. High Energy Phys. 04 (2021) 063.

[46] A. M. Galda, M. Neubert, and S. Renner, J. High Energy Phys. 06 (2021) 135.

[47] G. Alonso-Álvarez, F. Ertas, J. Jaeckel, F. Kahlhoefer, and L. J. Thormaehlen, J. High Energy Phys. 07 (2021) 059.

[48] B. W. Lee, C. Quigg, and H. B. Thacker, Phys. Rev. Lett. 38, 883 (1977).

[49] B. W. Lee, C. Quigg, and H. B. Thacker, Phys. Rev. D 16, 1519 (1977).

[50] M. S. Chanowitz, M. A. Furman, and I. Hinchliffe, Phys. Lett. B 78, 285 (1978).

[51] J. M. Cornwall, D. N. Levin, and G. Tiktopoulos, Phys. Rev. D 10, 1145 (1974); 11, 972(E) (1975).

[52] C. Bilchak, M. Kuroda, and D. Schildknecht, Nucl. Phys. B299, 7 (1988).

[53] G. J. Gounaris, J. Layssac, and F. M. Renard, Phys. Lett. B 332, 146 (1994).

[54] G. J. Gounaris, J. Layssac, J. E. Paschalis, and F. M. Renard, Z. Phys. C 66, 619 (1995).

[55] G. J. Gounaris, F. M. Renard, and G. Tsirigoti, Phys. Lett. B 350, 212 (1995).

[56] C. Degrande, EPJ Web Conf. 49, 14009 (2013).

[57] U. Baur and D. Zeppenfeld, Phys. Lett. B 201, 383 (1988).

[58] M. Dahiya, S. Dutta, and R. Islam, Phys. Rev. D 93, 055013 (2016).

[59] S. Ghosh, R. Islam, and A. Kundu, J. Phys. G 45, 015003 (2018).

[60] T. Corbett, O. J. P. Éboli, and M. C. Gonzalez-Garcia, Phys. Rev. D 91, 035014 (2015).

[61] T. Corbett, O. J. P. Éboli, and M. C. Gonzalez-Garcia, Phys. Rev. D 96, 035006 (2017).

[62] E. d. S. Almeida, O. J. P. Éboli, and M. C. Gonzalez-Garcia, Phys. Rev. D 101, 113003 (2020).

[63] W. J. Marciano, A. Masiero, P. Paradisi, and M. Passera, Phys. Rev. D 94, 115033 (2016).

[64] C. Cornella, P. Paradisi, and O. Sumensari, J. High Energy Phys. 01 (2020) 158.

[65] I. Brivio, M. B. Gavela, L. Merlo, K. Mimasu, J. M. No, R. del Rey, and V. Sanz, Eur. Phys. J. C 77, 572 (2017).

[66] W. A. Bardeen, S. H. H. Tye, and J. A. M. Vermaseren, Phys. Lett. 76B, 580 (1978).
[67] P. Di Vecchia and G. Veneziano, Nucl. Phys. B171, 253 (1980).

[68] G. G. di Cortona, E. Hardy, J. P. Vega, and G. Villadoro, J. High Energy Phys. 01 (2016) 034.

[69] R. S. Chivukula and H. Georgi, Phys. Lett. B 188, 99 (1987).

[70] L. J. Hall and L. Randall, Phys. Rev. Lett. 65, 2939 (1990).

[71] G. D'Ambrosio, G. F. Giudice, G. Isidori, and A. Strumia, Nucl. Phys. B645, 155 (2002).

[72] J. Bonilla, I. Brivio, M. B. Gavela, and V. Sanz, arXiv: 2107.11392.

[73] P. Draper and D. McKeen, Phys. Rev. D 85, 115023 (2012).

[74] M. Bauer, M. Neubert, and A. Thamm, J. High Energy Phys. 12 (2017) 044.

[75] M. Bauer, M. Heiles, M. Neubert, and A. Thamm, Eur. Phys. J. C 79, 74 (2019).

[76] H. Davoudiasl, R. Marcarelli, N. Miesch, and E. T. Neil, arXiv:2105.05866.

[77] J. C. Criado, Eur. Phys. J. C 79, 256 (2019).

[78] M. Jacob and G. C. Wick, Ann. Phys. (N.Y.) 7, 404 (1959).

[79] J. Jaeckel, M. Jankowiak, and M. Spannowsky, Phys. Dark Universe 2, 111 (2013).

[80] K. Mimasu and V. Sanz, J. High Energy Phys. 06 (2015) 173.

[81] J. Jaeckel and M. Spannowsky, Phys. Lett. B 753, 482 (2016).

[82] C. Frugiuele, E. Fuchs, G. Perez, and M. Schlaffer, J. High Energy Phys. 10 (2018) 151.

[83] N. Craig, A. Hook, and S. Kasko, J. High Energy Phys. 09 (2018) 028.

[84] J. Ebadi, S. Khatibi, and M. M. Najafabadi, Phys. Rev. D 100, 015016 (2019).

[85] C.-X. Yue, M.-Z. Liu, and Y.-C. Guo, Phys. Rev. D 100, 015020 (2019).

[86] M. B. Gavela, J. M. No, V. Sanz, and J. F. de Trocóniz, Phys. Rev. Lett. 124, 051802 (2020).

[87] S. C. İnan and A. V. Kisselev, J. High Energy Phys. 06 (2020) 183.

[88] G. Haghighat, D. H. Raissi, and M. M. Najafabadi, Phys. Rev. D 102, 115010 (2020).

[89] V. P. Goncalves and W. K. Sauter, Phys. Lett. B 811, 135981 (2020).

[90] A. Flórez, A. Gurrola, W. Johns, P. Sheldon, E. Sheridan, K. Sinha, and B. Soubasis, Phys. Rev. D 103, 095001 (2021).

[91] J. Bonilla, I. Brivio, J. Machado, and J. F. de Trocóniz (to be published).

[92] G. Aad et al. (ATLAS Collaboration), Phys. Rev. D 103, 112006 (2021).

[93] G. Aad et al. (ATLAS Collaboration), J. High Energy Phys. 02 (2021) 226. 